The University of Southern Mississippi

The Aquila Digital Community

Faculty Publications

$5-1-2017$

\title{
Comparative Analyses of Zebrafish Anxiety-Like Behavior Using Conflict-Based Novelty Tests
}

\author{
Elana V. Kysil \\ St. Petersburg State University \\ Darya A. Meshalkina \\ St. Petersburg State University \\ Erin E. Frick \\ University of Southern Mississippi, erin.frick@usm.edu \\ David J. Echevarria \\ University of Southern Mississippi, david.echevarria@usm.edu \\ Denis B. Rosemberg \\ The International Zebrafish Neuroscience Research Consortium
}

See next page for additional authors

Follow this and additional works at: https://aquila.usm.edu/fac_pubs

Part of the Psychiatry and Psychology Commons

\section{Recommended Citation}

Kysil, E. V., Meshalkina, D. A., Frick, E. E., Echevarria, D. J., Rosemberg, D. B., Maximino, C., Lima, M. G., Abreu, M. S., Giacomini, A. C., Barcellos, L. J., Song, C., Kalueff, A. V. (2017). Comparative Analyses of Zebrafish Anxiety-Like Behavior Using Conflict-Based Novelty Tests. Zebrafish, 14(3), 197-208.

Available at: https://aquila.usm.edu/fac_pubs/16664

This Article is brought to you for free and open access by The Aquila Digital Community. It has been accepted for inclusion in Faculty Publications by an authorized administrator of The Aquila Digital Community. For more information, please contact Joshua.Cromwell@usm.edu. 


\section{Authors}

Elana V. Kysil, Darya A. Meshalkina, Erin E. Frick, David J. Echevarria, Denis B. Rosemberg, Caio Maximino, Monica Gomes Lima, Murilo S. Abreu, Ana C. Giacomini, Leonardo J.G. Barcellos, Cai Song, and Allan V. Kalueff 


\section{Comparative analyses of zebrafish anxiety-like behavior using conflict-based novelty tests}

Elana V. Kysil ${ }^{1}$, Darya A. Meshalkina ${ }^{1}$, Erin E. Frick ${ }^{2}$, David J. Echevarria ${ }^{2,3}$, Denis B. Rosemberg ${ }^{3,4}$, Caio Maximino ${ }^{3,5,6}$, Monica Gomes Lima ${ }^{3,5,6}$, Murilo S. Abreu ${ }^{7}$, Ana C. Giacomini ${ }^{7}$, Leonardo J. G. Barcellos ${ }^{3,7,8}$, Cai Song ${ }^{9,10}$ and Allan V. Kalueff ${ }^{1,3,9,11,12^{*}}$

${ }^{1}$ Institute of Translational Biomedicine, St. Petersburg State University, St. Petersburg 199034, Russia;

${ }^{2}$ Department of Psychology, University of Southern Mississippi, Hattiesburg 39401, MS, USA;

${ }^{3}$ The International Zebrafish Neuroscience Research Consortium (ZNRC), Slidell 70458, LA, USA;

${ }^{4}$ Graduate Program in Biological Sciences: Toxicological Biochemistry, Federal University of Santa Maria. 1000 Roraima Avenue, Santa Maria 97105, RS, Brazil;

${ }^{5}$ Laboratory of Neurosciences and Behavior "Frederico Guilherme Graeff", Center for Biological and Health Sciences, Institute of Health and Biological Studies, Federal University of Southern and Southeastern Pará (UNIFESSPA), Marabá 68503, PA, Brazil;

${ }^{6}$ University of the State of Pará (UEPA), Marabá 68503, PA, Brazil;

${ }^{7}$ Postgraduate Program in Bio-Experimentation, University of Passo Fundo (UPF), Passo Fundo 99052, RS, Brazil;

${ }^{8}$ Postgraduate Program in Pharmacology, Federal University of Santa Maria (UFSM), Santa Maria 97105, RS, Brazil;

${ }^{9}$ Research Institute for Marine Drugs and Nutrition, College of Food Science and Technology, Guangdong Ocean University, Zhanjiang, 524025, Guangdong, China; 
${ }^{10}$ Graduate Institute of Neural and Cognitive Sciences, China Medical University Hospital, Taichung 40402, Taiwan;

${ }^{11}$ Ural Federal University, Ekaterinburg, 620002, Russia;

${ }^{12}$ ZENEREI Research Center, 309 Palmer Court, Slidell 70458, LA, USA

*Corresponding author:

Allan V. Kalueff, PhD,

Institute of Translational Biomedicine, St. Petersburg State University,

St. Petersburg 199034, Russia

Tel/Fax: +1-240-899-9571

Email: avkalueff@gmail.com 


\begin{abstract}
Modeling of stress and anxiety in adult zebrafish (Danio rerio) is increasingly utilized in neuroscience research and CNS drug discovery. Representing the most commonly used zebrafish anxiety models, the novel tank test (NTT) focuses on zebrafish diving in response to potentially threatening stimuli, whereas the light-dark test (LDT) is based on fish scototaxis (innate preference for dark vs. bright areas). Here, we systematically evaluate the utility of these two tests, combining meta-analyses of published literature with comparative in-vivo behavioral and whole-body endocrine (cortisol) testing. Overall, the NTT and LDT behaviors demonstrate a generally good cross-test correlation in-vivo, whereas meta-analyses of published literature shows that both tests have similar sensitivity to zebrafish anxiety-like states. Finally, NTT evokes higher levels of cortisol, likely representing a more stressful procedure than LDT. Collectively, our study reappraises NTT and LDT for studying anxietylike states in zebrafish, and emphasizes their developing utility for neurobehavioral research. These finding can help optimize drug screening procedures by choosing more appropriate models for testing anxiolytic or anxiogenic drugs.
\end{abstract}

Keywords: zebrafish; anxiety-like behavior; behavioral phenotyping; the novel tank test; the lightdark test 


\section{Introduction}

Evoked in response to novel, potentially dangerous situations, anxiety is a natural emotion which is critical for the organism to survive ${ }^{1,2}$. However, anxiety may also be pathological, appearing in inappropriate contexts and reaching high levels that disrupt normal life ${ }^{3}$. Anxiety disorders (ADs) are a diverse group of mental diseases defined by excessive worries or fears ${ }^{4}$. Widespread globally ${ }^{5}$, ADs represent the most prevalent psychiatric conditions, and may trigger other psychiatric disorders, such as depression and addiction ${ }^{6}$. Pathological anxiety is caused by aberrant 'emotionality' brain circuits, including the limbic system and cortex ${ }^{3,7}$. However, neural mechanisms of ADs remain poorly understood, necessitating novel experimental approaches and theoretical concepts ${ }^{8-11}$.

Animal experimental models, especially rodent paradigms, are an indispensable tool for understanding the basic neurobiology of ADs ${ }^{10,12}$. Reflecting the importance of translational, crossspecies analyses of neural phenotypes ${ }^{8,13-15}$, there is also a growing interest in widening the spectrum of model species in neurobehavioral research ${ }^{9,16-18}$. For example, adult zebrafish (Danio rerio) are rapidly emerging as a promising model organism to study anxiety- and other stress-related conditions 9, 19, 20. Among multiple behavioral tests and models ${ }^{21-25}$ (Table 1), the novel tank (NTT) and the light-dark (LDT) tests are the two most popular experimental paradigms of zebrafish anxiety ${ }^{26,27}$.

\subsection{The novel tank test (NTT)}

Based on geotaxis - an innate escape 'diving' behavior of fish in novel environments - the novel tank test (NTT) has long been used to assess adult zebrafish behaviors ${ }^{21}$ and drug responses ${ }^{20}$. Representing a conceptual analog of the rodent open field (OF) paradigm, NTT evokes motivational conflict between the 'protective' diving behavior and subsequent vertical exploration ${ }^{28}$. Indeed, when placed in novel environments, zebrafish initially spent more time at the bottom, reduce 'top' swimming, and exhibit more erratic movements and freezing/immobility episodes ${ }^{29}$. Later, due to habituation to the NTT novelty, zebrafish gradually explore the top area (potentially more dangerous for zebrafish in their natural habitats due to the presence of fish or bird predators). Stress and pharmacological agents can modulate these zebrafish NTT behaviors ${ }^{30,31}$, as anxiolytic drugs 
(e.g., buspirone ${ }^{32}$, chronic fluoxetine or diazepam ${ }^{33,34}$ ) tend to increase time spent in top, whereas anxiogenics promote diving, immobility and erratic movements ${ }^{31}$. Successfully applied to NTT, modern video-tracking techniques have markedly improved testing zebrafish behavior by reducing data processing time, increasing the ability to register more diverse parameters of locomotion as well as to analyze them off-line, objectively and simultaneously ${ }^{29,35-38}$.

Typically, the NTT apparatus consists of transparent narrow trapezoidal or rectangular tank divided into two equal halves either virtually ${ }^{39}$ or with horizontal line marked directly on the wall 40. The parameters analyzed in NTT target two major phenotypic domains - the exploration and locomotion ${ }^{41}$. The main NTT anxiety-related endpoints are time spent in the upper/bottom zone, the latency to enter the top, the number of crossings between the zones, as well as the number and duration of freezing (a total absence of movement, except for the gills and eyes) and erratic movements (sharp changes in direction and velocity) ${ }^{40,42}$. Locomotor phenotypes can be assessed by recording various automated zebrafish NTT endpoints, including distance traveled, absolute or average turn angle, average and maximal swimming speed, meandering or the number of $360^{\circ}$ rotations ${ }^{29}$. Reduced vertical NTT exploration may also represent inhibited swimming activity in the presence of sedative agents (e.g., high sedative concentrations of ethanol) and not necessarily reflect increased anxiety-like states ${ }^{43}$. Conversely, a greater exploration of the top can be due to enhanced locomotion, a phenotype usually observed after exposure to psychostimulant or hallucinogenic drugs 44-46. Therefore, evaluating more than one endpoint is needed to judge whether the effect is locomotor or anxio-tropic. Thus, there is a great value in assessing locomotor parameters of zebrafish during novelty stress in NTT using automated video tracking software and 3D reconstruction plots of behavior as neurophenotyping tools ${ }^{35,47}$.

Zebrafish also demonstrate behavioral differences in the NTT depending on their strain, sex, age $^{27,48}$ and housing conditions ${ }^{49}$. For example, fish exposed to ethanol show mild hyperlocomotion in the home tank water, but display increased anxiety-like behavior with unaltered locomotion in newly replaced water ${ }^{50}$. The apparatus size and shape also modulate fish behavioral responses in this model, as zebrafish constantly housed in a narrow tank identical to the NTT show no NTT-specific 
behaviors during testing, including absent diving response or changes in swimming speed ${ }^{32}$. However, the main stimuli controlling zebrafish NTT diving responses remain unclear ${ }^{51}$. In the wild, zebrafish feeds in the water column, and therefore their vertical distribution is a trade-off between feeding and predator avoidance ${ }^{52}$. While exposure to fish predators or their models may unalter zebrafish bottom-dwelling ${ }^{35,53-55}$, presenting a computer-animated image of a bird silhouette strongly increases their diving response ${ }^{56}$. Moreover, when fish are exposed to a tank with two compartments differing in real and perceived depth, the preference is observed for the side allowing further escape from the surface, but not the side with closer proximity to the substrate ${ }^{57}$, collectively suggesting that NTT diving is an escape from the water surface rather than approaching the bottom.

\subsection{The light-dark test (LDT)}

The LDT is also widely used in zebrafish, since adult fish avoid brightly lit areas and spend more time in the dark ${ }^{22,58}$. This behavior is associated with the natural tendency of wild zebrafish to show overt scototaxis, thereby facilitating crypsis (avoidance the detection by other animals). Since adequate response to external stimuli is crucial for animal survival, zebrafish light avoidance rises from morning to evening, but decreases at night ${ }^{59}$. Various stressors (e.g., ${ }^{60}$ ) or anxiogenic substances (e.g., ${ }^{26,61}$ ) predictably increase time spent in dark, allowing the LDT to evaluate anxietyrelated behaviors and drugs. Other main endpoints used in this test are the number of total transitions between the two compartments, latency to enter the white area, the number of risk assessment episodes (fast entries to the lit area followed by re-entries to the dark, or as partial entries to the white), thigmotaxis in the white area, and other behavioral endpoints (e.g., erratic swimming and freezing) similar to those recorded in the NTT ${ }^{62}$.

The predominantly accepted interpretation of LDT is that adult zebrafish show robust dark preference in the test (note, however, that zebrafish larvae have reversed light preference ${ }^{63,64}$ ). A typical LDT apparatus is a rectangular glass or acryl tank consisting of two equal vertical portions: black and white or black and transparent chambers ${ }^{44,65,66}$. The color of the lighter portion (white vs. transparent) can change preference, as animals tend to prefer the transparent vs. the black compartment ${ }^{65,67}$. Another modification of the preference paradigm apparatus is the light dark plus 
maze with transparent walls and black or white arms' floor coloration ${ }^{68}$. Size of the light/dark box also usually differs markedly between the laboratories. Parts of the preference tank can be divided with a grey divider (after habituation, fish are able to swim between light and dark areas freely without a sliding door) ${ }^{69,70}$ or have no physical barrier ${ }^{71,72}$. Considering that the currently available software tools do not properly detect animal in the black compartments, their behaviors are usually recorded in the light chamber, or using a gray floor (which may per se influence the behavior). Like for the NTT, the stimulus control is not fully established in the LDT ${ }^{51}$. For example, varying light levels or color of the white compartment and intra-/inter-session habituation data suggest that zebrafish LDT behavior is not driven solely by white aversion (photophobia) or scototaxis, but is based on approachavoidance conflict ${ }^{51}$. As a result, it remains unclear whether the LDT and NTT may target different aspects of anxiety-like behavior, or different levels of anxiety-like states, or both. Here, we systematically compare these commonly used aquatic tests and evaluate their utility in characterizing zebrafish behavioral syndromes.

\section{Materials and Methods}

\subsection{Behavioral meta-analyses}

Although the question of data comparison and testing priority was already raised for NTT and LDT 51,73 , their systematic comparative analyses have not been performed. Addressing this knowledge gap here, we first analyzed the literature, focusing on NTT and LDT studies of various anxiolytic, anxiogenic and toxic substances (Table 2). The inclusion criteria required 1) both tests be used in the same study available in PubMed; 2) testing the same concentrations of the drug(s); and 3) group size, mean and SEM/SD values be indicated for each group. Because of multiple parameters measured in NTT and LDT, we chose two most frequently measured endpoints that can also be considered functionally analogous: the time spent in, and the number of transitions to, the top half of NTT or the lit half of the LDT, respectively. For these endpoints, we calculated Standardized Mean Difference (SMD, Fig. 1) commonly used for the effect size estimation in meta-analyses ${ }^{74}$. As some mean and SEM for SMD calculations were represented in the graphs in the original publications, we performed their accurate quantification using the ImageJ software ${ }^{75}$. Statistical analysis was 
conducted using the metafor package ${ }^{76}$ for $\mathrm{R}$ version $3.2 .5^{77}$. We used a mixed-effects model using assay as a moderator to indicate a possible advantage of using the NTT or the LDT. While it is possible that treatment differences (i.e., drug, concentration/dose, etc.) are important moderators, we were interested in the main effect of any treatment on the behavioral endpoints, and in differences between the NTT and LDT. Therefore, using assay as a moderator, such as in our present analyses, was expected to be able to uncover differences in sensitivity between the two tests (see further).

\subsection{Cortisol responses to NTT and LDT procedures}

Complementing behavioral endpoints, various physiological biomarkers are indispensable for clinical and preclinical $\mathrm{AD}$ research ${ }^{78-80}$. In a separate in-vivo experiment we directly compared physiological consequences of the two tests, using ELISA assays to access whole-body cortisol levels in adult zebrafish following their acute single NTT or LDT exposure ${ }^{40}$. Briefly, a total of 45 adult zebrafish ( 50/50 male/female ratio) of the wild-type short-fin strain were housed 1 fish/L in $20-\mathrm{L}$ tanks equipped with biological filters at the University of Passo Fundo (Passo Fundo, Brazil), under constant aeration and a 14-h light:10 h dark photoperiod. Water temperature was maintained at $27 \pm$ $0.3{ }^{\circ} \mathrm{C}$; with pH kept at $7.0 \pm 0.05$, dissolved oxygen kept at $6.0 \pm 0.05 \mathrm{mg} / \mathrm{L}$, total ammonia at $<0.01$ $\mathrm{mg} / \mathrm{L}$, total hardness at $6 \mathrm{mg} / \mathrm{L}$, and alkalinity at $22 \mathrm{mg} / \mathrm{L} \mathrm{CaCO}_{3}$. The experiment utilized three groups of fish: controls (experimentally naïve, unexposed fish), NTT- and LDT-exposed fish (which remained in their tests for $10 \mathrm{~min}$, and were then immediately sacrificed for cortisol analysis using ELISA). Fish were gently transferred individually (using the net) from their hometanks to the testing apparatus (NTT or LDT) for $10 \mathrm{~min}$. The NTT represented a glass tank $24 \times 8 \times 20 \mathrm{~cm}$ (width $\times$ depth $\times$ height ${ }^{60}$. The LDT apparatus consisted of a glass tank $(18 \times 9 \times 7 \mathrm{~cm}$; width $\times$ depth $\times$ high $)$ divided by a sliding guillotine-type partition $(9 \times 7 \mathrm{~cm})$ in two equally sized dark and white compartments, filled with water ${ }^{60}$. Fish were individually placed in the light zone of the apparatus, and evaluated for 10 min. Behavioral results were subjected to one-way ANOVA, and were further analyzed by Tukey post-hoc test to compare all three groups (Fig. 3). The two tests were further compared between themselves by the U-test (Fig. 3).

\subsection{Correlational analyses of behaviors generated in NTT and LDT}


Commonly used in neurobehavioral research, correlational analyses were next applied to the NTT and LDT data collected from a large cohort of male and female wild-type zebrafish housed in Federal University of Sul e Sudeste do Para (Maraba, Brazil). While the test battery effects were reported as minimal for zebrafish ${ }^{27}$, the testing order was randomized. Since animals were kept in single groups, no sequence generation method was used. The animals came from different tanks, and were randomly drawn. Similar to ${ }^{81}$, correlations were taken between measurements of time and transitions for each test. Time on top and time on white constituted a first set of "operational definitions" of anxiety-like behavior in theses assays, and transitions to top and transitions to white constituted the second set of operational definitions. Overall, we found convergence for the first set of operational definitions ("monotrait-heteromethod", marked by A in Table 3), as the measures moderately but significantly correlated. The second set of operational definitions revealed a strong significant correlation, supporting the possibility that both measures in the two tests reflect the similar/overlapping behavioral trait. Another type of correlation ("heterotrait-monomethod", marked by $\mathrm{B}$ in Table 3) refers to the relationship between the different operational definitions of the same trait measured using the same method, and for both assays, were non-significant. The last type of correlation ("heterotrait-heteromethod", $\mathrm{C}$ in Table 3) examined to the relationship between activity in one assay and occupancy of the less protected area in the other.

\section{Results}

\subsection{NTT and LDT sensitivity to stress and CNS drugs: behavioral meta-analyses}

The omnibus meta-analysis performed here indicated an effect of drugs/treatments on the time spent in the top/lit part (Fig. 1), as the intercept for the mixed-effects model was significant $(\beta=$ 1.0923$, CI 95\%[0.2241, 1.9604]; $\mathrm{z}=2.446, \mathrm{p}=0.0137)$ but the test for the moderators was not $\left(\mathrm{QM}_{(\mathrm{df}}\right.$ $=1)=0.0204, p=0.8865)$. This suggests that in the specific dataset of drugs and treatments acting on different targets, the NTT and the LDT remained equally sensitive to the treatments if the experimental conditions (e.g., concentrations) were the same ( $\beta=-0.04, \mathrm{CI} 95 \%[-0.5899,0.5098]$; z $=-0.1428, \mathrm{p}=0.8865)$. The lack of significance was also characteristic for comparisons in the drug groups, although effects differed for LDT and NTT when transitions were considered (Fig. 1), since 
treatments increased transitions to white in LDT $(\beta=4.67$, CI 95\% $[2.44,6.9], z=4.1051, \mathrm{p}<0.0001)$ but not to top in NTT $(\beta=-3.85$, CI 95\% $[-9.11,1.42], \mathrm{z}=-1.4325, \mathrm{p}=0.1520)$. The omnibus test revealed a significant effect of the moderators $\left(\mathrm{QM}_{(\mathrm{df}=1)}=9.1854, \mathrm{p}=0.0024\right)$, with a significant assay effect $(\mathrm{z}=3.0307, \mathrm{p}=0.0024)$. Analyzing SMDs between the two endpoints themselves

revealed some controversy in the effect direction (e.g., for fluoxetine or pCPA ${ }^{34}$ ). As expected for not including treatment as a moderator, the heterogeneity of the results was significantly high $\left(\tau^{2}=\right.$ $\left.1.8160 \pm 0.2886 ; \mathrm{I}^{2}=89.1 \%, \mathrm{QE}_{(\mathrm{df}=104)}=747.6830, \mathrm{p}<0.0001\right)$. Although the lack of significant SMD differences between the most frequently measured parameter (time in aversive portions) was unexpected, it suggests that none of the two tests produces higher effect sizes on these parameters, thus making the two tests complementary, rather interchangeable. Finally, funnel plot analysis (Fig. 2) also indicates considerable publication bias towards significant findings, which should be considered and, eventually, corrected in future research.

\subsection{Cortisol responses to NTT and LDT}

Overall, while both tests were more stressful vs. control, NTT was significantly more stressful than LDT, based on test-evoked cortisol responses (Fig. 3). This result is important and predictable, given the rigorous nature of NTT diving responses vs. a more 'protective' LDT choice situation. Moreover, this also suggests that while the measures of bottom and dark preference in these two paradigms can be conceptually similar, they may also differ in the levels of evoked stress and/or in their ability to act as tests (i.e., to measure different behaviors). Clearly, further studies are needed to examine the underlying stress responses and their neural circuitry in both models.

\subsection{Correlations between behaviors generated in NTT and LDT}

Overall, correlations between transitions to white and time on top were not significant in this analysis, whereas correlations between transitions to top and time in white were significant, but smaller than the monotrait-heteromethod correlations (Table 3). Thus, correlations in the "validity diagonal" A were higher than the heterotrait-heteromethod and heterotrait-monomethod correlations, which reflect convergent and discriminant validity ${ }^{82}$. Overall, these results support the notion that 
the operational definitions of anxiety in LDT and NTT seem to converge on the same zebrafish behavioral trait.

\section{Discussion}

Despite the advantages of using fish for modeling mental disorders, all animal models are limited since they cannot fully recapitulate the complex repertoire of human behavior ${ }^{17}$. Furthermore, it can be difficult to distinguish between various subtypes of animal anxiety (e.g., generalized anxiety vs. fear), and they have not yet been dissected in zebrafish ${ }^{51}$. In addition, despite shared construct rationale and targeting similar evolutionarily-conserved traits in fish and rodents ${ }^{19}$, test- and speciesdifferences may further complicate data interpretation and analyses. One example is 3D tracking of zebrafish movement ${ }^{35,83}$ in NTT (which enables quantification of vertical geotaxic anxiety-like response), but is impossible to assess in 2D-based OF (focusing on thigmotaxis instead). On the one hand, LDT typically measures a limited number of behavioral endpoints, enabling a more focused characterization of anxiety-related phenotypes. However, LDT does not usually track zebrafish in 3D, and therefore misses endpoints related to angular velocity or turn angle. Thus, while NTT may have an added value of registering more behavioral endpoints and phenomena, LDT can be quite useful for its high-throughput and lower stress. Furthermore, as many drugs modifying anxiety display characteristic U-shaped dose-response curve for commonly used endpoints ${ }^{84}$, the range of concentrations used for drug screening should be sufficient to account for possible effect increase and dropdown. Clearly, this may slow down the screening process, but will help minimize type II errors in CNS drug discovery.

Another aspect that merits further consideration is the extent of automation that can be achieved using these approaches, and whether this represents a drawback, especially given the unrivalled potential of zebrafish for high-throughput analysis. Indeed, while without the potential for automation, there may be strong between-lab reliability for these measures, other extraneous factors (such as housing/husbandry and testing procedures) may trigger such variance as well. Thus, we strongly support further automation in studies, and welcome their further inclusion in subsequent meta-analyses, in addition to offering a clear experimental advantage of assessing reliably a wider 
range of behavioral analysis in a shorter period of time. Likewise, another factor that is largely overlooked in zebrafish literature is the differences in basal locomotor responses by individual fish, and how this may affect the typical responses behavioral phenotypes. While individual differences in zebrafish behaviors do exist, this aspect was not in the scope of our study, and was somewhat mitigated here by using relatively large n's in our cohorts. Nevertheless, we fully recognize the importance of individual differences in zebrafish NTT and LDT behaviors, and acknowledge the fact that automation of fish testing may not only reduce 'phenotypic noise' by better controlling the testing environments, but can also help better detect meaningful patterns in individual differences for multiple computer-generated parameters.

In summary, as NTT and LDT remain widely used tests of zebrafish anxiety, they are often considered 'similar' in their construct, face and predictive validity. Analyzing their effect sizes across multiple studies, including data from our own group, we found their comparable sensitivity, based on similar SMD values for their main endpoints. Thus, the two tests can be both needed for assessing zebrafish anxiety-like behaviors and drug screening, reinforcing Prof. Slava Lapin's famous notion "one experiment is not an experiment, and one behavioral test is not a test". However, although the cumulative responsivity of NTT and LDT to treatments appear similar, some drugs may affect one test more than the other. Respectively, this suggests that predictive validity of the two models may be somewhat different (Fig. 1). For example, as NTT also measures endpoints that are also relevant to other behavioral domains (e.g., locomotion or cognition/habituation), it has the advantage of versatility and the ability to characterize $3 \mathrm{D}$ locomotion ${ }^{35,71,83}$. At the same time, given our endocrine/cortisol data (Fig. 3), it is likely that NTT also represents a more stressful procedure than LDT. Accordingly, this may contribute to the two tests' differing predictive validity, as anxiogenic drugs may have floor effects in NTT, which can therefore be more useful for testing anxiolytics instead. In contrast, a seemingly 'less aversive' LDT may be more suitable for testing anxiogenics, but may exhibit more ceiling effects when screening anxiolytic drugs. Likewise, it is possible to suggest that the two models may differ in their ability to reflect decision-making behavior. For example, a more survival-driven innate 'diving' response may seem to be more forced upon the 
animal by the NTT testing protocol, as compared to a less aversive LDT procedure, which affords the fish more time and a better control over their light-dark preference behaviors (e.g., see ${ }^{85}$ ). Quite interesting, this hypothesis merits further scrutiny, and may help develop novel behavioral models beyond affective domains (e.g., targeting zebrafish impulsivity based on their NTT vs. LDT responses).

Further analyses of the exact nature of stress in the two aquatic tests remains an important priority for zebrafish behavioral research ${ }^{86}$, because they are both related to anxiety, but may differ in the domains of stress, fear, aversion, visual comprehension and decision-making. It may also require studies of brain activation patterns in one or another setup with paralleling the results to the mammalian brain studies. Such analyses have already been performed in LDT ${ }^{85}$, implicating the medial zone of the dorsal telencephalic region and the dorsal nucleus of the ventral telencephalic area (the teleost homologs of the mammalian amygdala and striatum, respectively) in fish anxiety responses. It would therefore be interesting to conduct a similar study in NTT. Future dissection of the differences between brain activation patterns may necessitate sophisticated in-vivo imaging studies in freely moving animals, which can be particularly useful since the genetically encoded calcium reporters can be analyzed in transparent strains of zebrafish.

Finally, with a large number of behavioral endpoints resulting from zebrafish behavioral tests (e.g., ${ }^{35,87,88}$ ), the integral parameter that would consistently reflect the level of zebrafish anxiety, is still missing. Would it help to have such a biomarker? Clearly yes, since, for example, some of NTT and LDT endpoints are closely and directly related to anxiety and endocrine (cortisol) levels, and can have higher weight in the integral anxiety testing. Thus, the upcoming aim of zebrafish behavioral neuroscience can be to rank various endpoints for the overall effect appraisal, and to extract important biological information from those various ranked phenotypes accordingly. In other words, this may involve creating an integral index based on multiple factors and endpoints (somewhat similar to a credit score in financial world) to provide a rough estimate of fish anxiety levels, thereby empowering time/cost-efficient drug screening. One attempt to establish such an integral value has been made recently ${ }^{89}$, using the concept of Integrated Biological Response that summarizes the range of 
biomarkers into single star plot, that is then analyzed by square and shape. Such plots are rather visual and allow for input endpoints ranking and at the same time they can be expressed in the form of single number, enabling rapid comparison. However, a conceptually different (but equally fruitful) approach can be to continue to explore the potential complexity of zebrafish anxiety behavior, based on a theoretical possibility (not yet tested empirically) that zebrafish anxiety-like states may include subtypes, similar to clinical generalized anxiety- vs. fear/panic-like states, and the respective rodent analogous models that do distinguish these states ${ }^{90-94}$. At the same time, it may also be possible ${ }^{17,84}$ that zebrafish affective phenotypes (and their respective circuits) are merely not as complicated as in mammals, and may reflect broader (e.g., generalized anxiety-like) affective categories rather than target more specific AD subtypes, such as anxiety vs. fear/panic.

\section{Acknowledgements:}

The study was coordinated through the International Zebrafish Neuroscience Research Consortium (ZNRC), and this collaboration was supported by St. Petersburg State University Intramural Research program (DMM, EVK, AVK), Ural Federal University (AVK), Guangdong Ocean University (CS, AVK), the University of Passo Fundo (LJGB), CNPq grant 470260/2013 (LJGB) and CNPq research fellowships 301992/2014-2 (LJGB) and 307595/2015-3 (DBR). The funders had no involvement in the study design, data collection or analysis, and manuscript preparation. AVK is the Chair of ZNRC, and his research is supported by the Russian Foundation for Basic Research (RFBR) grant 16-04-00851. The authors thank Mr Rodrigo Zanandrea (University of Passo Fundo, Brazil) for his assistance with zebrafish cortisol analyses. 
Figure 1. Meta-analysis results for effects of various drugs/treatments on zebrafish novel tank test (NTT) and light-dark test (LDT) behaviors, including the number of transitions (A) and time spent $(B)$ in respective top or light areas. Data are presented as forest plots (based on 27 studies listed in Table 1 that use both behavioral tests) for the standardized mean differences (SMD) for treated vs. control groups with corresponding $95 \%$ confidence intervals in the individual studies, based on a mixed-effects model.

Figure 2. The funnel plot showing the relationships between residual values vs. standard errors (estimated by the mixed-effects model) in data presented in Fig. 1; bands refer to confidence intervals at the $90 \%$ (white area), $95 \%$ (light gray area), and 99\% (dark grey area) levels.

Figure 3. Whole-body cortisol levels in control vs. NTT- and LDT-tested fish assessed immediately after behavior test procedure ( $n=15$ in each group). There was significant treatment effect by one-way ANOVA $\left(\mathrm{F}_{2,41}=13.14 ; \mathrm{P}<0.0001\right) ; * \mathrm{P}<0.05$, $* * * * \mathrm{P}<0.0001$ for Tukey post-hoc test (left panel). The two tests also significantly differed between themselves (P $<0.05$, U-test, right panel). 


\begin{tabular}{|c|c|c|}
\hline Test/Model & Details & References \\
\hline \multicolumn{3}{|c|}{ Tests (used to assess anxiety levels) } \\
\hline Open field test & $\begin{array}{l}\text { A direct analog of rodent open field, this paradigm measures mainly horizontal } \\
\text { locomotion and thigmotaxis. The apparatus can have diverse size, shape and } \\
\text { color. Endpoints in this test include the time spent in the periphery or center of } \\
\text { the tank (s), distance traveled in each zone in the tank }(\mathrm{m}) \text {, number of transitions } \\
\text { between zones, velocity in each zone of the tank }(\mathrm{m} / \mathrm{s}) \text {, number of freezing bouts } \\
\text { and time spent frozen }(\mathrm{s})\end{array}$ & $24,41,44$ \\
\hline $\begin{array}{l}\text { Social } \\
\text { preference test }\end{array}$ & $\begin{array}{l}\text { The test has been applied to evaluate zebrafish response to con- and } \\
\text { heterospecifics. Zebrafish social preference may be altered because of the effect } \\
\text { of anxiety-related pharmaceutical substances or environmental stressors. }\end{array}$ & 95,96 \\
\hline Shoaling & $\begin{array}{l}\text { Fish are placed to the test apparatus in groups and impact of stressor or } \\
\text { pharmacological manipulation are assessed measuring alterations in shoal } \\
\text { cohesion (it is higher when zebrafish feel anxiety). }\end{array}$ & 23,97 \\
\hline $\begin{array}{l}\text { Predator } \\
\text { avoidance test }\end{array}$ & $\begin{array}{l}\text { The test examines fear- and anxiety-like behavior in the presence of a natural } \\
\text { stressor. Zebrafish individually or in group are placed in one part of apparatus } \\
\text { with two separate compartments, another arm contains predator (e.g., Indian leaf } \\
\text { fish, Nandus nandus). }\end{array}$ & $55,98,99$ \\
\hline $\begin{array}{l}\text { Predator } \\
\text { exposure test }\end{array}$ & $\begin{array}{l}\text { The test is aimed at evaluation of fear- and anxiety-like behavior following a } \\
\text { brief exposure to a natural predator (e.g. Indian leaf fish), its image or robotic } \\
\text { models }\end{array}$ & 100 \\
\hline $\begin{array}{l}\text { Boldness and } \\
\text { novel object } \\
\text { approaching }\end{array}$ & $\begin{array}{l}\text { During the novel object test zebrafish either individually or in groups are placed } \\
\text { to a cylindric tank and, after period of acclimatization, the unfamiliar object is } \\
\text { added to the apparatus. Main endpoints include the latency to approach the object } \\
\text { (s), frequency of approach, time spent near (within } 1.5 \text { body-lengths) the object } \\
\text { (s), number of freezing bouts, and time spent frozen (s). }\end{array}$ & 25,101 \\
\hline $\begin{array}{l}\text { Emergence } \\
\text { test }\end{array}$ & $\begin{array}{l}\text { This test assesses anxiety behaviors by placing animals in a reassuring chamber, } \\
\text { and measuring latency to exit the chamber. }\end{array}$ & 102 \\
\hline $\begin{array}{l}\text { Food } \\
\text { neophobia }\end{array}$ & $\begin{array}{l}\text { Avoidance of novel food, which can be assessed by measuring latency to } \\
\text { approach novel food items, and frequency/duration of time spent interacting with } \\
\text { novel food items }\end{array}$ & 103 \\
\hline \multicolumn{3}{|c|}{ Models (used to evoke anxiety-like states) } \\
\hline Beaker stress & $\begin{array}{l}\text { During beaker stressor protocols, an individual fish is separated from its shoal } \\
\text { and confined in a } 250-\mathrm{ml} \text { beaker filled with } 100-\mathrm{ml} \text { of house tank water. This } \\
\text { stressor markedly increases baseline cortisol levels after } 15 \text {-min exposure. }\end{array}$ & 17 \\
\hline $\begin{array}{l}\text { Impoverished } \\
\text { housing }\end{array}$ & $\begin{array}{l}\text { Housing fish singly in a barren environment increases anxiety-like behaviors in } \\
\text { the NTT and LDT }\end{array}$ & 49 \\
\hline Chronic stress & $\begin{array}{l}\text { Pathological anxiety is commonly seen in zebrafish exposed to various chronic } \\
\text { stress models }\end{array}$ & 33,104 \\
\hline $\begin{array}{l}\text { Social } \\
\text { isolation }\end{array}$ & Chronic social isolation decreases, while acute isolation increases anxiety levels & $17,51,105,106$ \\
\hline $\begin{array}{l}\text { Genetic } \\
\text { models }\end{array}$ & Various inbred and outbred zebrafish strains with different anxiety levels & 20,86 \\
\hline
\end{tabular}


Table 2. List of treatments and publications used for the analysis.

\begin{tabular}{|c|c|c|}
\hline Treatment & Dose $(\mathrm{mg} / \mathrm{L})$, other details & References \\
\hline \multicolumn{3}{|l|}{ Anxiolytic-like treatments } \\
\hline 2,5-Dimethoxy-4-bromoamphetamine (DOB) & $0.05,0.1,0.5,1$ & 107 \\
\hline para-Methoxyamphetamine (PMA) & $0.0005,0.005,0.05,0.1,0.25,0.5$ & 107 \\
\hline Buspirone & 25,50 & 34 \\
\hline \multirow[t]{2}{*}{ Fluoxetine } & $2.5,5,10$ & 34 \\
\hline & $5 \mathrm{mg} / \mathrm{kg}$ long-fin and leopard strains & 108 \\
\hline para-chlorophenylalanine (pCPA) & 300 twice & 34 \\
\hline WAY 100635 & $0.003,0.03$ & 34 \\
\hline \multirow[t]{2}{*}{ Ethanol (acute) } & $0.5,1,1.5 \%$ & 72 \\
\hline & $0.5 \%$ & 68 \\
\hline Desipramine & 25 & 68 \\
\hline Nicotine & 25 & 68 \\
\hline Chlordiazepoxide & 25 & 68 \\
\hline Citalopram & 100 & 68 \\
\hline Taurine & $42,150,400$ & 89 \\
\hline Piracetam & 200 (chronic, for 7 days) & 109 \\
\hline $\mathrm{N}$-acetylcysteine & $0.1,1,10$ & 60 \\
\hline \multicolumn{3}{|l|}{ Hallucinogenic-like treatments } \\
\hline 3,4-Methylenedioxymethamphetamine (MDMA)* & $2.5,5,10$ & 107 \\
\hline Lysergic acid diethylamide (LSD)* & 0.25 & 44 \\
\hline Ibogaine* & 10,20 & 71 \\
\hline \multicolumn{3}{|l|}{ Anxiogenic-like treatments } \\
\hline SB 224289 & $2.5,5$ & 34 \\
\hline $1 \%$ Ethanol (chronic) withdrawal & 2,6 days $(1,7$ days for LDT) & 72 \\
\hline Alarm substance & Short-fin, leopard strains & 61 \\
\hline Yohimbine & 25 & 68 \\
\hline \multicolumn{3}{|l|}{ Toxic treatments } \\
\hline Paraquat & 20 & 110 \\
\hline Methylmercury & $1,5 \mu \mathrm{g} / \mathrm{g}$ & 62 \\
\hline Dimethyl sulfoxide (DMSO)* & $0.05 \%$ & 68 \\
\hline Copper & 0.006 & 111 \\
\hline
\end{tabular}

* The drug also exerts anxiolytic-like effects in zebrafish 
Table 3. Cross-correlations between occupancy (time on white, time on top) and locomotion (entries on white, entries on top) measurements in $\mathbf{4 0}$ adult wild-type control zebrafish tested in the novel tank (NTT) and light-dark (LDT) tests at the Federal University do Sul e Sudeste do Pará, Maraba, Brazil. Values represent Pearson's correlation coefficients based on individual scores across all animals. (A) Convergence of operational definitions; (B) different operational definitions, same method; (C) different operational definitions, different methods (see text for details).

\begin{tabular}{|llll|}
\hline Behaviors/Tests & NTT time & NTT transitions & LDT time \\
\hline NTT transitions & (B) 0.08 & (C) $\mathbf{0 . 4 3}$ & \\
LDT time & (A) 0.55 & (A) $\mathbf{0 . 7 5}$ & (B) 0.18 \\
LDT transitions & (C) 0.11 & & \\
\hline
\end{tabular}




\section{References}

1. Campos AC, Fogaca MV, Aguiar DC, Guimaraes FS: Animal models of anxiety disorders and stress. Rev Bras Psiquiatr. 2013;35 Suppl 2:S101-11.

2. Crocq MA: A history of anxiety: from Hippocrates to DSM. Dialogues Clin Neurosci. 2015;17:319-25.

3. Nuss P: Anxiety disorders and GABA neurotransmission: a disturbance of modulation. Neuropsychiatr Dis Treat. 2015;11:165-75.

4. American Psychiatric Association: Diagnostic and Statistical Manual of Mental Disorders. 5 ed. Arlington: American Psychiatric Publishing.; 2013.

5. Baxter AJ, Scott KM, Vos T, Whiteford HA: Global prevalence of anxiety disorders: a systematic review and meta-regression. Psychol Med. 2013;43:897-910.

6. Remes $\mathrm{O}$, Brayne $\mathrm{C}$, van der Linde R, Lafortune L: A systematic review of reviews on the prevalence of anxiety disorders in adult populations. Brain Behav. 2016;6:e00497.

7. Etkin A: Functional neuroanatomy of anxiety: a neural circuit perspective. Curr Top Behav Neurosci. 2010;2:251-77.

8. Stewart AM, Kalueff AV: Developing better and more valid animal models of brain disorders. Behavioural brain research. 2015;276:28-31.

9. Stewart AM, Ullmann JF, Norton WH, Parker MO, Brennan $\mathrm{CH}$, Gerlai R, et al.: Molecular psychiatry of zebrafish. Mol Psychiatry. 2015;20:2-17.

10. Fraser LM, Brown RE, Hussin A, Fontana M, Whittaker A, O'Leary TP, et al.: Measuring anxiety- and locomotion-related behaviours in mice: a new way of using old tests. Psychopharmacology. 2010;211:99112.

11. Adamec R, Burton P, Blundell J, Murphy DL, Holmes A: Vulnerability to mild predator stress in serotonin transporter knockout mice. Behavioural brain research. 2006;170:126-40.

12. Griebel G, Holmes A: 50 years of hurdles and hope in anxiolytic drug discovery. Nature reviews Drug discovery. 2013;12:667-87.

13. Stewart AM, Kalueff AV: Anxiolytic drug discovery: what are the novel approaches and how can we improve them? Expert opinion on drug discovery. 2014;9:15-26.

14. de Mooij-van Malsen AJ, Pjetri E, Kas MJ: The genetic and epigenetic landscape for CNS drug discovery targeting cross-diagnostic behavioral domains. Eur J Pharmacol. 2015;753:135-9.

15. Kas MJ, Glennon JC, Buitelaar J, Ey E, Biemans B, Crawley J, et al.: Assessing behavioural and cognitive domains of autism spectrum disorders in rodents: current status and future perspectives. Psychopharmacology. 2014;231:1125-46.

16. Kalueff AV, Stewart AM, Gottesman, II: Rethinking CNS disorders: time for new drug targets? Trends Pharmacol Sci. 2014;35:491-2.

17. Kalueff AV, Echevarria DJ, Stewart AM: Gaining translational momentum: more zebrafish models for neuroscience research. Progress in neuro-psychopharmacology \& biological psychiatry. 2014;55:1-6.

18. Stewart AM, Yang E, Nguyen M, Kalueff AV: Developing zebrafish models relevant to PTSD and other trauma- and stressor-related disorders. Prog Neuropsychopharmacol Biol Psychiatry. 2014;55:67-79. 19. Kalueff AV, Stewart AM, Gerlai R: Zebrafish as an emerging model for studying complex brain disorders. Trends in pharmacological sciences. 2014;35:63-75.

20. Stewart AM, Braubach O, Spitsbergen J, Gerlai R, Kalueff AV: Zebrafish models for translational neuroscience research: from tank to bedside. Trends in neurosciences. 2014;37:264-78.

21. Levin ED, Bencan Z, Cerutti DT: Anxiolytic effects of nicotine in zebrafish. Physiology \& behavior. 2007;90:54-8.

22. Maximino C, Marques de Brito T, Dias CA, Gouveia A, Jr., Morato S: Scototaxis as anxiety-like behavior in fish. Nat Protoc. 2010;5:209-16.

23. Miller N, Gerlai R: Quantification of shoaling behaviour in zebrafish (Danio rerio). Behav Brain Res. 2007;184:157-66.

24. Stewart A, Cachat J, Wong K, Gaikwad S, Gilder T, DiLeo J, et al.: Homebase behavior of zebrafish in novelty-based paradigms. Behavioural processes. 2010;85:198-203.

25. Wright D, Rimmer LB, Pritchard VL, Krause J, Butlin RK: Inter and intra-population variation in shoaling and boldness in the zebrafish (Danio rerio). Naturwissenschaften. 2003;90:374-7. 
26. Maximino C, da Silva AW, Gouveia A, Jr., Herculano AM: Pharmacological analysis of zebrafish (Danio rerio) scototaxis. Progress in neuro-psychopharmacology \& biological psychiatry. 2011;35:624-31. 27. Song C, Yang L, Wang J, Chen P, Li S, Liu Y, et al.: Building neurophenomics in zebrafish: Effects of prior testing stress and test batteries. Behav Brain Res. 2016;311:24-30.

28. Stewart A KF, DiLeo J, Min Chung K, Cachat J, Goodspeed J, et al.: The developing utility of zebrafish in modeling neurobehavioral disorders. Int J Comp Psychol. 2010:104-21.

29. Cachat J, Stewart A, Grossman L, Gaikwad S, Kadri F, Chung KM, et al.: Measuring behavioral and endocrine responses to novelty stress in adult zebrafish. Nature protocols. 2010;5:1786-99.

30. Cachat J, Canavello P, Elegante M, Bartels B, Hart P, Bergner C, et al.: Modeling withdrawal syndrome in zebrafish. Behavioural brain research. 2010;208:371-6.

31. Vignet C, Begout ML, Pean S, Lyphout L, Leguay D, Cousin X: Systematic screening of behavioral responses in two zebrafish strains. Zebrafish. 2013;10:365-75.

32. Bencan Z, Sledge D, Levin ED: Buspirone, chlordiazepoxide and diazepam effects in a zebrafish model of anxiety. Pharmacol Biochem Behav. 2009;94:75-80.

33. Giacomini AC, Abreu MS, Giacomini LV, Siebel AM, Zimerman FF, Rambo CL, et al.: Fluoxetine and diazepam acutely modulate stress induced-behavior. Behav Brain Res. 2016;296:301-10.

34. Maximino C, Puty B, Benzecry R, Araujo J, Lima MG, de Jesus Oliveira Batista E, et al.: Role of serotonin in zebrafish (Danio rerio) anxiety: relationship with serotonin levels and effect of buspirone, WAY 100635, SB 224289, fluoxetine and para-chlorophenylalanine (pCPA) in two behavioral models. Neuropharmacology. 2013;71:83-97.

35. Cachat J, Stewart A, Utterback E, Hart P, Gaikwad S, Wong K, et al.: Three-dimensional neurophenotyping of adult zebrafish behavior. PloS one. 2011;6:e17597.

36. Delcourt J, Becco C, Vandewalle N, Poncin P: A video multitracking system for quantification of individual behavior in a large fish shoal: advantages and limits. Behav Res Methods. 2009;41:228-35.

37. Fontaine $\mathrm{E}$, Lentink D, Kranenbarg S, Muller UK, van Leeuwen JL, Barr AH, et al.: Automated visual tracking for studying the ontogeny of zebrafish swimming. J Exp Biol. 2008;211:1305-16.

38. Stewart AM, Grieco F, Tegelenbosch RA, Kyzar EJ, Nguyen M, Kaluyeva A, et al.: A novel 3D method of locomotor analysis in adult zebrafish: Implications for automated detection of CNS drug-evoked phenotypes. J Neurosci Methods. 2015;255:66-74.

39. Wang Y, Li S, Liu W, Wang F, Hu LF, Zhong ZM, et al.: Vesicular monoamine transporter 2 (Vmat2) knockdown elicits anxiety-like behavior in zebrafish. Biochem Biophys Res Commun. 2016;470:792-7. 40. Egan RJ, Bergner CL, Hart PC, Cachat JM, Canavello PR, Elegante MF, et al.: Understanding behavioral and physiological phenotypes of stress and anxiety in zebrafish. Behavioural brain research. 2009;205:38-44.

41. Stewart AM, Gaikwad S, Kyzar E, Kalueff AV: Understanding spatio-temporal strategies of adult zebrafish exploration in the open field test. Brain research. 2012;1451:44-52.

42. Wang J, Wang X, Xiong C, Liu J, Hu B, Zheng L: Chronic bisphenol A exposure alters behaviors of zebrafish (Danio rerio). Environ Pollut. 2015;206:275-81.

43. Rosemberg DB, Braga MM, Rico EP, Loss CM, Cordova SD, Mussulini BH, et al.: Behavioral effects of taurine pretreatment in zebrafish acutely exposed to ethanol. Neuropharmacology. 2012;63:613-23.

44. Grossman L, Utterback E, Stewart A, Gaikwad S, Chung KM, Suciu C, et al.: Characterization of behavioral and endocrine effects of LSD on zebrafish. Behavioural brain research. 2010;214:277-84.

45. Seibt KJ, da Luz Oliveira R, Rosemberg DB, Savio LE, Scherer EB, Schmitz F, et al.: MK-801 alters Na+, $\mathrm{K}+-\mathrm{ATPase}$ activity and oxidative status in zebrafish brain: reversal by antipsychotic drugs. J Neural Transm. 2012;119:661-7.

46. Neelkantan N, Mikhaylova A, Stewart AM, Arnold R, Gjeloshi V, Kondaveeti D, et al.: Perspectives on zebrafish models of hallucinogenic drugs and related psychotropic compounds. ACS chemical neuroscience. 2013;4:1137-50.

47. Stewart AM, Gerlai R, Kalueff AV: Developing highER-throughput zebrafish screens for in-vivo CNS drug discovery. Frontiers in behavioral neuroscience. 2015:in press.

48. Lopez Patino MA, Yu L, Yamamoto BK, Zhdanova IV: Gender differences in zebrafish responses to cocaine withdrawal. Physiology \& behavior. 2008;95:36-47.

49. Collymore C, Tolwani RJ, Rasmussen S: The Behavioral Effects of Single Housing and Environmental Enrichment on Adult Zebrafish (Danio rerio). Journal of the American Association for Laboratory Animal Science : JAALAS. 2015;54:280-5. 
50. Tran S, Facciol A, Gerlai R: Home tank water versus novel water differentially affect alcohol-induced locomotor activity and anxiety related behaviours in zebrafish. Pharmacol Biochem Behav. 2016;144:13-9.

51. Maximino C. BR, Broock Rosemberg D., Batista E.: A comparison of the light/dark and novel tank tests in zebrafish. Behaviour. 2012;149(10-12):1099-123.

52. Parichy DM: Advancing biology through a deeper understanding of zebrafish ecology and evolution. Elife. 2015;4.

53. Blaser R, Gerlai R: Behavioral phenotyping in zebrafish: comparison of three behavioral quantification methods. Behav Res Methods. 2006;38:456-69.

54. Gerlai R, Fernandes Y, Pereira T: Zebrafish (Danio rerio) responds to the animated image of a predator: towards the development of an automated aversive task. Behav Brain Res. 2009;201:318-24. 55. Bass SL, Gerlai R: Zebrafish (Danio rerio) responds differentially to stimulus fish: the effects of sympatric and allopatric predators and harmless fish. Behav Brain Res. 2008;186:107-17.

56. Luca RM, Gerlai R: Animated bird silhouette above the tank: acute alcohol diminishes fear responses in zebrafish. Behavioural brain research. 2012;229:194-201.

57. Blaser RE GK: Depth preference in zebrafish, Danio rerio: Control by surface and substrate cues. Anim Behav 2012:953-9.

58. Hascoet M, Bourin M, Nic Dhonnchadha BA: The mouse light-dark paradigm: a review. Prog Neuropsychopharmacol Biol Psychiatry. 2001;25:141-66.

59. Wang J, Liu C, Ma F, Chen W, Liu J, Hu B, et al.: Circadian clock mediates light/dark preference in zebrafish (Danio rerio). Zebrafish. 2014;11:115-21.

60. Mocelin R, Herrmann AP, Marcon M, Rambo CL, Rohden A, Bevilaqua F, et al.: N-acetylcysteine prevents stress-induced anxiety behavior in zebrafish. Pharmacol Biochem Behav. 2015;139 Pt B:121-6.

61. Quadros VA, Silveira A, Giuliani GS, Didonet F, Silveira AS, Nunes ME, et al.: Strain- and contextdependent behavioural responses of acute alarm substance exposure in zebrafish. Behav Processes. 2016;122:1-11.

62. Maximino C, Araujo J, Leao LK, Grisolia AB, Oliveira KR, Lima MG, et al.: Possible role of serotoninergic system in the neurobehavioral impairment induced by acute methylmercury exposure in zebrafish (Danio rerio). Neurotoxicology and teratology. 2011;33:727-34.

63. Bai Y, Liu H, Huang B, Wagle M, Guo S: Identification of environmental stressors and validation of light preference as a measure of anxiety in larval zebrafish. BMC Neurosci. 2016;17:63.

64. Steenbergen PJ, Richardson MK, Champagne DL: Patterns of avoidance behaviours in the light/dark preference test in young juvenile zebrafish: a pharmacological study. Behav Brain Res. 2011;222:15-25. 65. Champagne DL, Hoefnagels CC, de Kloet RE, Richardson MK: Translating rodent behavioral repertoire to zebrafish (Danio rerio): relevance for stress research. Behav Brain Res. 2010;214:332-42. 66. Stewart A MC, de Brito TM, Herculano AM, Gouveia Jr A, Morato S, et al.: Neurophenotyping of adult zebrafish using the light/dark box paradigm. Zebrafish neurobehavioral

protocols. 2011:p. 157-67.

67. Gerlai R, Lahav M, Guo S, Rosenthal A: Drinks like a fish: zebra fish (Danio rerio) as a behavior genetic model to study alcohol effects. Pharmacology, biochemistry, and behavior. 2000;67:773-82.

68. Sackerman J, Donegan JJ, Cunningham CS, Nguyen NN, Lawless K, Long A, et al.: Zebrafish Behavior in Novel Environments: Effects of Acute Exposure to Anxiolytic Compounds and Choice of Danio rerio Line. International journal of comparative psychology / ISCP ; sponsored by the International Society for Comparative Psychology and the University of Calabria. 2010;23:43-61.

69. Magno LD, Fontes A, Goncalves BM, Gouveia A, Jr.: Pharmacological study of the light/dark preference test in zebrafish (Danio rerio): Waterborne administration. Pharmacol Biochem Behav. 2015;135:169-76.

70. Mansur Bde M, Dos Santos BR, Dias CA, Pinheiro Mde S, Gouveia A, Jr.: Effects of the number of subjects on the dark/light preference of Zebrafish (Danio rerio). Zebrafish. 2014;11:560-6.

71. Cachat J, Kyzar EJ, Collins C, Gaikwad S, Green J, Roth A, et al.: Unique and potent effects of acute ibogaine on zebrafish: the developing utility of novel aquatic models for hallucinogenic drug research. Behavioural brain research. 2013;236:258-69.

72. Mathur $P$, Guo S: Differences of acute versus chronic ethanol exposure on anxiety-like behavioral responses in zebrafish. Behavioural brain research. 2011;219:234-9.

73. Blaser RE, Rosemberg DB: Measures of anxiety in zebrafish (Danio rerio): dissociation of black/white preference and novel tank test. PloS one. 2012;7:e36931. 
74. Higgins JPT, \& Green, S.: Cochrane Handbook for Systematic Reviews of Interventions Version 5.1.0 2011.

75. Schindelin J, Rueden CT, Hiner MC, Eliceiri KW: The ImageJ ecosystem: An open platform for biomedical image analysis. Mol Reprod Dev. 2015;82:518-29.

76. Viechtbauer W: Conducting meta-analyses in $\mathrm{R}$ with the metafor package. Journal of Statistical Software. 2010:1-48.

77. R Core Team: R: A Language and Environment for Statistical Computing. 3.2.5 ed. R Foundation for Statistical Computing, Vienna, Austria, 2016.

78. Abreu MS, Koakoski G, Ferreira D, Oliveira TA, Rosa JG, Gusso D, et al.: Diazepam and fluoxetine decrease the stress response in zebrafish. PloS one. 2014;9:e103232.

79. Ussher M, West R, Evans P, Steptoe A, McEwen A, Clow A, et al.: Reduction in cortisol after smoking cessation among users of nicotine patches. Psychosomatic medicine. 2006;68:299-306.

80. Dinan TG, Majeed T, Lavelle E, Scott LV, Berti C, Behan P: Blunted serotonin-mediated activation of the hypothalamic-pituitary-adrenal axis in chronic fatigue syndrome. Psychoneuroendocrinology.

1997;22:261-7.

81. van der Staay FJ, Kerbusch S, Raaijmakers W: Genetic correlations in validating emotionality. Behav Genet. 1990;20:51-62.

82. Campbell D.T. FDW: Convergent and discriminant validation by the

multitrait-multimethod matrix. Psychol Bulletin. 1959;56:81-105.

83. Maaswinkel $\mathrm{H}$, Zhu L, Weng $\mathrm{W}$ : Using an automated 3D-tracking system to record individual and shoals of adult zebrafish. Journal of visualized experiments : JoVE. 2013:50681.

84. Stewart A, Gaikwad S, Kyzar E, Green J, Roth A, Kalueff AV: Modeling anxiety using adult zebrafish: a conceptual review. Neuropharmacology. 2012;62:135-43.

85. Lau BY, Mathur P, Gould GG, Guo S: Identification of a brain center whose activity discriminates a choice behavior in zebrafish. Proceedings of the National Academy of Sciences of the United States of America. 2011;108:2581-6.

86. Stewart AM, Kaluyeva AA, Poudel MK, Nguyen M, Song C, Kalueff AV: Building Zebrafish Neurobehavioral Phenomics: Effects of Common Environmental Factors on Anxiety and Locomotor Activity. Zebrafish. 2015;12:339-48.

87. Maximino C, da Silva AW, Araujo J, Lima MG, Miranda V, Puty B, et al.: Fingerprinting of psychoactive drugs in zebrafish anxiety-like behaviors. PloS one. 2014;9:e103943.

88. Stewart AM, Cachat J, Green J, Gaikwad S, Kyzar E, Roth A, et al.: Constructing the habituome for phenotype-driven zebrafish research. Behavioural brain research. 2013;236:110-7.

89. Mezzomo NJ, Silveira A, Giuliani GS, Quadros VA, Rosemberg DB: The role of taurine on anxiety-like behaviors in zebrafish: A comparative study using the novel tank and the light-dark tasks. Neurosci Lett. 2016;613:19-24.

90. Pearson BL, Defensor EB, Blanchard DC, Blanchard RJ: Applying the ethoexperimental approach to neurodevelopmental syndrome research reveals exaggerated defensive behavior in Mecp2 mutant mice. Physiol Behav. 2015;146:98-104.

91. Belzung C: Rodent models of anxiety-like behaviors: are they predictive for compounds acting via non-benzodiazepine mechanisms? Curr Opin Investig Drugs. 2001;2:1108-11.

92. Carboni E, Wieland S, Lan NC, Gee KW: Anxiolytic properties of endogenously occurring pregnanediols in two rodent models of anxiety. Psychopharmacology (Berl). 1996;126:173-8.

93. Rotzinger S, Lovejoy DA, Tan LA: Behavioral effects of neuropeptides in rodent models of depression and anxiety. Peptides. 2010;31:736-56.

94. Holmes A, Yang RJ, Lesch KP, Crawley JN, Murphy DL: Mice lacking the serotonin transporter exhibit 5-HT(1A) receptor-mediated abnormalities in tests for anxiety-like behavior. Neuropsychopharmacology : official publication of the American College of Neuropsychopharmacology. 2003;28:2077-88.

95. Barba-Escobedo PA, Gould GG: Visual social preferences of lone zebrafish in a novel environment: strain and anxiolytic effects. Genes Brain Behav. 2012;11:366-73.

96. Saverino C, Gerlai R: The social zebrafish: behavioral responses to conspecific, heterospecific, and computer animated fish. Behav Brain Res. 2008;191:77-87.

97. Engeszer RE, Ryan MJ, Parichy DM: Learned social preference in zebrafish. Curr Biol. 2004;14:881-4.

98. Gerlai R: Antipredatory behavior of zebrafish: adaptive function and a tool for translational

research. Evol Psychol. 2013;11:591-605. 
99. Gerlai R: Zebrafish antipredatory responses: a future for translational research? Behav Brain Res. 2010;207:223-31.

100. Cianca V, Bartolini T, Porfiri M, Macri S: A robotics-based behavioral paradigm to measure anxietyrelated responses in zebrafish. PloS one. 2013;8:e69661.

101. Moretz JA, Martins, E.I.P., et al.: Behavioral syndromes and the evolution of correlated behavior in zebrafish. Behav Ecol. 2007;18 (3):556-62.

102. Toms CN, Echevarria DJ: Back to basics: searching for a comprehensive framework for exploring individual differences in zebrafish (danio rerio) behavior. Zebrafish. 2014;11:325-40.

103. Kalueff AV, Gebhardt M, Stewart AM, Cachat JM, Brimmer M, Chawla JS, et al.: Towards a comprehensive catalog of zebrafish behavior 1.0 and beyond. Zebrafish. 2013;10:70-86.

104. Marcon M, Herrmann AP, Mocelin R, Rambo CL, Koakoski G, Abreu MS, et al.: Prevention of unpredictable chronic stress-related phenomena in zebrafish exposed to bromazepam, fluoxetine and nortriptyline. Psychopharmacology (Berl). 2016;233:3815-24.

105. Shams S, Chatterjee D, Gerlai R: Chronic social isolation affects thigmotaxis and whole-brain serotonin levels in adult zebrafish. Behav Brain Res. 2015;292:283-7.

106. Parker MO, Millington ME, Combe FJ, Brennan $\mathrm{CH}$ : Housing conditions differentially affect physiological and behavioural stress responses of zebrafish, as well as the response to anxiolytics. PLoS One. 2012;7:e34992.

107. Ponzoni L, Sala M, Braida D: Ritanserin-sensitive receptors modulate the prosocial and the anxiolytic effect of MDMA derivatives, DOB and PMA, in zebrafish. Behav Brain Res. 2016;314:181-9.

108. Maximino C, Puty B, Matos Oliveira KR, Herculano AM: Behavioral and neurochemical changes in the zebrafish leopard strain. Genes, brain, and behavior. 2013;12:576-82.

109. Grossman L, Stewart A, Gaikwad S, Utterback E, Wu N, Dileo J, et al.: Effects of piracetam on behavior and memory in adult zebrafish. Brain research bulletin. 2011;85:58-63.

110. Nunes ME, Muller TE, Braga MM, Fontana BD, Quadros VA, Marins A, et al.: Chronic Treatment with Paraquat Induces Brain Injury, Changes in Antioxidant Defenses System, and Modulates Behavioral Functions in Zebrafish. Mol Neurobiol. 2016.

111. Haverroth GM, Welang C, Mocelin RN, Postay D, Bertoncello KT, Franscescon F, et al.: Copper acutely impairs behavioral function and muscle acetylcholinesterase activity in zebrafish (Danio rerio). Ecotoxicol Environ Saf. 2015;122:440-7. 